CUBO A Mathematical Journal

Vol.12, Noㅡ, (7r-96). June 2010

\title{
Generalized solutions of the Cauchy problem for the Navier-Stokes system and diffusion processes
}

\author{
S. Albeverio \\ Institut für Angewandte Mathematik, Universität Bonn, \\ Wegelerstr. 6, D-53115 Bonn, Germany \\ SFB 611,HCM, Bonn, BiBoS, Bielefeld - Bonn, \\ CERFIM, Locarno and USI (Switzerland) \\ email: albeverio@uni-bonn.de \\ and \\ YA. BELOPOLSKAYA \\ St.Petersburg State University for Architecture and Civil Engineering, \\ 2-ja Krasnoarmejskaja 4, 190005, St.Petersburg, Russia \\ email: yana@yb1569.spb.edu
}

\begin{abstract}
We reduce the construction of a weak solution of the Cauchy problem for the Navier-Stokes system to the construction of a stochastic problem solution. Under suitable conditions we solve the stochastic problem and prove that simultaneously we obtain a weak (generalized) solution to the Cauchy problem for the Navier-Stokes system.
\end{abstract}

\section{RESUMEN}

Nosotros reducimos la construcción de una solución débil de un problema de Cauchy para el sistema de Navier-Stokes para la construcción de la resolución de un problema estocástico. Bajo condiciones convenientes resolvimos el problema estocástico y probamos que simultáneamente obtenemos una solución débil (generalizada) para el problema de Cauchy del sistema de Navier-Stokes. 
Key words and phrases: Stochastic flows, diffusion processes, nonlinear parabolic equations, Cauchy problem.

AMS Subj. Class.: 60H10, 60J60 , 35G05, 35K45

\section{Introduction}

The main purpose of this article is to construct both strong and weak solutions (in certain functional classes) of the Cauchy problem for the Navier-Stokes (N-S) system

$$
\begin{gathered}
\frac{\partial u}{\partial t}+(u, \nabla) u=\nu \Delta u-\nabla p, \quad u(0, x)=u_{0}(x), \quad x \in R^{3} \\
\operatorname{div} u=0 .
\end{gathered}
$$

Here $u(t, x) \in R^{3}, x \in R^{3}, t \in[0, \infty)$ is the velocity of the fluid at the position $x$ at time $t$ and $\nu>0$ is the viscosity coefficient. $p(t, x)$ is a scalar field called the pressure which appears in the equation to enforce the incompressibility condition (1.2). There exists a number of papers [1] - [4] and others where the system (1.1), (1.2) was treated from the probabilistic point of view on the base of stochastic models.

In particular in our previous paper [1] the system (1.1), (1.2) was reduced to a probabilistic problem presented in the form of the following system of equations

$$
\begin{gathered}
d \xi(\tau)=-u(t-\tau, \xi(\tau)) d \tau+\sigma d w(\tau) \\
u(t, x)=E_{0, x}\left[u_{0}(\xi(t))+\int_{0}^{t} \nabla p(t-\tau, \xi(\tau)) d \tau\right] \\
p(t, x)=2 E\left[\int_{0}^{\infty} \gamma(t, x+B(t)) d t\right]=2 E\left[\int_{0}^{\infty} \operatorname{tr}[\nabla u]^{2}(t, x+B(t)) d t\right] .
\end{gathered}
$$

Here $\sigma=\sqrt{2 \nu}, w(t)$ and $B(t)$ are independent standard Wiener processes valued in $R^{3}, \operatorname{Tr}[\nabla u]^{2}=$ $\sum_{i, k=1}^{3} \nabla_{i} u_{k} \nabla_{k} u_{i}$. It was shown in [1] that if the initial value $u_{0}$ is a $C^{3}$ - function the functions $u(t, x), p(t, x)$ given by (1.4), (1.5) are $C^{2+\alpha}$ solutions of (1.1), (1.2) for $0<\alpha<1$.

In the present paper we consider an alternative probabilistic system which allows to construct a weak (distributional) solution to (1.1), (1.2). The approach developed here is based on the theory of stochastic flows due to Kunita [5], [6] and the results due to Belopolskaya and Dalecky [7], [8].

The article is composed as follows. In section 2 we give some preliminary information concerning different analytical approaches to the notion of a solution of the Navier-Stokes system. Here we recall some common ways to exclude the pressure and to obtain a closed equation for the velocity, introduce necessary functional spaces and state various notions of solutions to (1.1), (1.2). In section 3 we state our approach and prove main results. In the last section we compare our approach and results with the Euler-Lagrange approach to incompressible fluids developed by Constantin and Iyer [9],[10].

\section{Preliminaries}

Within a classical approach to the N-S system one excludes the pressure from $(1.1),(1.2)$ and investigates the resulting nonlinear pseudo-differential equation. To this end first one can derive formally 
from (1.1),(1.2) the relation

$$
-\Delta p(t, x)=\gamma(t, x)
$$

where

$$
\gamma(t, x)=\sum_{k, j=1}^{3} \nabla_{k} u_{j} \nabla_{j} u_{k}=\operatorname{Tr}[\nabla u]^{2}=\nabla \cdot \nabla \cdot u \otimes u .
$$

Then given an $R^{3}$-valued vector field $u(t)$ over $R^{3}$ the operator $\mathbf{P}$ is defined by

$$
\mathbf{P} u(t)=u(t)-\nabla \Delta^{-1} \nabla \cdot u(t) .
$$

Here and below $u \cdot v$ denotes the inner product in $R^{3}$ of the vectors $u$ and $v$.

The map $\mathbf{P}$ called the Leray projection is a projection of the space $\mathbf{L}^{2}\left(R^{3}\right) \equiv L^{2}\left(R^{3}\right)^{3}$ of square integrable vector fields to the space of divergence free vector fields.

Since the formal solution of the Poisson equation (2.1) is given by

$$
p=\Delta^{-1} \gamma=\Delta^{-1} \nabla \cdot \nabla \cdot u \otimes u
$$

one can present $\nabla p$ in the form

$$
\nabla p=\nabla \Delta^{-1} \nabla \cdot \nabla \cdot u \otimes u
$$

keeping in mind that $\operatorname{div} u=0$. Substituting this expression for $\nabla p$ into (1.1) one obtains the following Cauchy problem

$$
\frac{\partial u}{\partial t}=\nu \Delta u-\mathbf{P} \nabla \cdot(u \otimes u), \quad u(0)=u_{0} .
$$

When (2.5) is solved then the pressure is reconstructed from the Poisson equation (2.1).

The Leray projection $\mathbf{P}$ is used to solve the N-S system both in numerous analytical papers (see, e.g., [11] for references) and in papers where the N-S system is studied from the probabilistic point of view $[2],[3],[10]$. In this paper we avoid the direct application of the Leray projection and construct the solution of (1.1), (1.2) via stochastic processes associated with (1.1) and (2.1).

To give a rigorous definition of a solution for the N-S system we have to specify the required functional spaces.

Let $\mathcal{D}=\mathcal{D}\left(R^{3}\right)=C_{c}^{\infty}$ denote the space of all infinitely differentiable real valued functions on $R^{3}$ with compact support equipped with the Schwartz topology and let $\mathcal{D}^{\prime}$ be its topological dual. Let $\langle\phi, \psi\rangle=\int_{R^{3}} \phi(x) \psi(x) d x$ denote the natural coupling between $\phi \in \mathcal{D}$ and $\psi \in \mathcal{D}^{\prime}$. If it will not lead to misunderstandings we will use the same notation for vector fields $u$ and $v$ as well, that is

$$
\langle h, u\rangle=\int_{R^{3}} \sum_{k=1}^{3} h_{k}(x) u_{k}(x) d x .
$$

Let $\mathbf{D}\left((0, T) \times R^{3}\right)=\left(\mathcal{D}^{\prime}\left((0, T) \times R^{3}\right)\right)^{3}$ denote the space of $R^{3}$-valued vector fields $h$ with components $h_{k} \in \mathcal{D}$ and $\mathbf{D}^{\prime}$ denote the space dual to $\mathbf{D}\left(R^{3}\right)$.

The Leray weak solution of the N-S system on $[0, T] \times R^{3}$ is a vector field $u(t, x)$ in $\left(\mathcal{D}^{\prime}((0, T) \times\right.$ $\left.\left.R^{3}\right)\right)^{3}$ such that $u$ is locally square integrable on $(0, T) \times R^{3}$, satisfies $\operatorname{div} u=0$ and there is a distribution $p \in \mathcal{D}^{\prime}\left((0, T) \times R^{3}\right)$ such that

$$
\frac{\partial u}{\partial t}=\nu \Delta u-\nabla \cdot(u \otimes u)-\nabla p, \quad \lim _{t \rightarrow 0} u(t)=u_{0}
$$


holds in the sense of distributions.

The Kato mild solution is a solution $u$ to the following integral equation

$$
u(t)=e^{t \Delta} u_{0}-\int_{0}^{t} e^{(t-s) \Delta} \mathbf{P} \nabla \cdot(u \otimes u)(s) d s .
$$

Note that instead of looking for $u(t, x)$ and $p(t, x)$ one may look for their Fourier images $\hat{u}(\lambda)=$ $(2 \pi)^{-\frac{3}{2}} \int_{R^{3}} e^{-i \lambda \cdot x} u(x) d x$. The Leray and Kato approaches stated in original terms and in terms of the Fourier transformation of the Navier-Stokes system were developed in a number of papers (see, e.g., references in the book by Lemarie-Rieusset [11]).

Below we will need as well the following functional spaces:

the space $C\left(R^{d}, R^{n}\right)$ of bounded continuous functions mapping $R^{d}$ to $R^{n}$,

the space $C\left(R^{3}, R^{1}\right)=C\left(R^{3}\right)$ of bounded continuous real functions $f$ with the norm $\|f\|_{\infty}=$ $\sup _{x \in R^{3}}|f(x)|$;

the space $\mathbf{C}\left(R^{3}\right)$ of bounded continuous vector functions with the norm $\|u\|_{\infty}=\sup _{x \in R^{3}}\|u(x)\|$, where $\|\cdot\|$ is the norm in $R^{3}$;

the space $\mathbf{C}_{0}\left(R^{3}\right)$ of continuous vector functions with compact supports; the Banach space $\mathbf{L}^{q}\left(R^{3}\right)$ of integrable functions $f$ with norm $\|f\|_{q}=\left(\int_{R^{3}}\|f(x)\|^{q} d x\right)^{\frac{1}{q}}$;

the space $\mathbf{C}^{k}\left(R^{3}\right)$ of k-times differentiable functions with the norm $\|g\|_{C^{k}}=\sum_{|\beta| \leq k}\left\|D^{\beta} g\right\|_{\infty}$;

the space $\mathbf{C}^{k, \alpha}\left(R^{3}\right)$ (for a natural number $k$ ) of vector fields whose $\mathrm{k}$-th derivatives are Hölder continuous with exponent $\alpha, 0<\alpha \leq 1$ with norm

$$
\|g\|_{C^{k, \alpha}}=\|g\|_{C^{k}}+[g]_{k+\alpha}
$$

and

$$
[g]_{k+\alpha}=\sum_{|\beta|=k} \sup _{x, y \in R^{3}} \frac{\left|D^{\beta} g(x)-D^{\beta} g(y)\right|}{|x-y|^{\alpha}} .
$$

Let $Z$ denote the set of all integers, and suppose that $k \in Z$ is positive and $1<q<\infty$. Denote by $W^{k, q}=W^{k, q}\left(R^{3}\right)$ the set of all real functions $h$ defined on $R^{3}$ such that $h$ and all its distributional derivatives $\nabla^{\alpha} h$ of order $|\alpha|=\sum \alpha_{j} \leq k$ belong to $L^{q}\left(R^{3}\right)$. It is a Banach space with norm

$$
\|h\|_{k, p}=\left(\sum_{|\alpha| \leq k} \int_{R^{3}}\left|D^{\alpha} h(x)\right|^{q} d x\right)^{\frac{1}{q}} .
$$

Denote by $W_{0}^{k, q}$ the subspace of functions from $W^{k, q}=W^{k, q}\left(R^{3}\right)$ with compact supports.

Finally we will need some spaces of locally integrable functions. Let $G \subset R^{3}$ be a bounded domain, $p$ be a positive integer and $f: G \rightarrow R^{1}$ be a Lebesgue measurable function. The set of functions $\left\{f: \int_{K}|f(x)|^{p} d x<\infty\right.$ for all compact subsets $\left.K \subset G\right\}$ is denoted by $L_{l o c}^{p}$ and called a space of $p$ - locally integrable functions. Although $L_{l o c}^{p}(G)$ are not normed spaces they are readily topologized. Namely a sequence $\left\{u_{n}\right\}$ converges to $u$ in $L_{l o c}^{p}(G)$ if $\left\{u_{n}\right\} \rightarrow u$ in $L^{p}(K)$ for each open $K$ having compact closure in $G$ and $\|u\|_{p, l o c}=\left(\int_{K}\|u(x)\|^{p} d x\right)^{\frac{1}{p}}<\infty$.

In a natural way one can define the spaces $\mathbf{W}^{\mathbf{k}, \mathbf{q}}$ and $\mathbf{L}_{l o c}^{p}(G)$ of vector fields with components in $W^{k, p}$ and in $L_{l o c}^{p}(G)$. 


\section{A probabilistic approach to the Navier-Stokes system}

Let us come back to the Navier-Stokes system written in the form

$$
\begin{gathered}
\frac{\partial u}{\partial t}+(u, \nabla) u=\frac{\sigma^{2}}{2} \Delta u-\nabla p, \quad u(0, x)=u_{0}(x), \quad x \in R^{3} \\
-\Delta p=\gamma
\end{gathered}
$$

with $\gamma$ defined by (1.3).

Our main purpose in this section is to construct a diffusion process that allows us to obtain a a weak solution to (3.1), (3.2) via its probabilistic representation. To be more precise we intend to reduce the system (3.1), (3.2) to a certain system of stochastic equations and to construct its solution. Then we have to verify that in this way we have constructed a weak solution of (3.1), (3.2).

As above let $w(t), B(t)$ be standard $R^{3}$-valued independent Wiener processes defined on a probability space $(\Omega, \mathcal{F}, P)$. Given a bounded measurable function $f(x)$ and a stochastic process $\xi(t)$ we denote $E_{s, x} f(\xi(t)) \equiv E f\left(\xi_{s, x}(t)\right)$ the conditional expectation under the condition $\xi(s)=x$.

Given a function $g(t, x) \in R^{3}$, a smooth (in $x$ ) function $q(t, x) \in R^{1}, t \in(0, \infty), x \in R^{3}$ and a constant $\sigma$ we consider stochastic processes $\xi^{g}(t)$ and $\lambda(t)$ satisfying the stochastic equations

$$
\begin{gathered}
d \xi_{y}^{g}(t)=g\left(t, \xi_{y}^{g}(t)\right) d t-\sigma d w(t), \quad \xi_{y}^{g}(0)=y \in R^{3}, \\
\lambda(t)=u_{0}-\int_{0}^{t} \nabla q\left(\tau, \phi_{0, \tau}^{g}\right) d \tau,
\end{gathered}
$$

where $\phi_{0, t}^{g}$ denotes the stochastic map in $R^{3}$ generated by the process $\xi^{g}(t), \phi_{0, t}^{g}(y)=\xi_{y}^{g}(t)$. The map $\phi_{0, t}^{g}: R^{3} \rightarrow R^{3}$ is called a stochastic flow.

The processes $\xi^{g}(t)$ and $\lambda(t)$ are auxiliary ones. The main role in our considerations is played by the stochastic flow $\psi_{t, 0}$ which is an inverse flow to $\phi_{0, t}, \psi_{t, 0}\left(\phi_{0, t}(y)\right)=y$. To construct the flow $\psi_{t, 0}$ we need the process $\hat{w}(\theta)=w(t-\theta)-w(t)$ which is proved to be the standard Wiener process.

Here we use the results of the Kunita theory of stochastic flows [5],[6] and extend them to the case of stochastic processes associated with nonlinear PDEs.

Actually we consider the closed system

$$
\begin{gathered}
d \psi_{t, \theta}(x)=-u\left(\theta, \psi_{t, \theta}(x)\right) d \theta+\sigma d \hat{w}(\theta), \quad \psi_{t, t}(x)=x, \\
u(t, x)=E\left[u_{0}\left(\psi_{t, 0}(x)\right)-\int_{0}^{t} \nabla p\left(\tau, \psi_{t, \tau}(x)\right) d \tau\right], \\
-2 \nabla p(t, x)=E\left[\int_{0}^{\infty} \frac{1}{\tau} \gamma(t, x+B(\tau)) B(\tau) d \tau\right],
\end{gathered}
$$

where $\gamma$ is given by (1.3) and look for a solution $u(t, x), p(t, x), \psi_{t, \theta}(x)$ of this system under some assumptions on the initial data $u_{0}$ to be specified below.

To construct the solution of (3.4) - (3.6) we consider its differential prolongation. Namely, we consider the following formal relation

$$
d \eta^{x}(\theta)=-\nabla u\left(\theta, \psi_{t, \theta}(x)\right) \eta^{x}(\theta) d \theta, \quad \eta^{x}(t)=I,
$$


where $I$ is the identity matrix acting in $R^{3}$, and one of two formal relations for $\nabla u(t, x)$

$$
\nabla u(t, x)=E\left[\nabla u_{0}\left(\psi_{t, 0}(x)\right) \eta^{x}(t)-\int_{0}^{t} \nabla^{2} p\left(\tau, \psi_{t, \tau}(x)\right) \eta^{x}(\tau) d \tau\right] .
$$

or

$$
\nabla u(t, x)=E\left[\nabla u_{0}\left(\psi_{t, 0}(x)\right) \eta^{x}(t)-\int_{0}^{t} \frac{\nabla p\left(\tau, \psi_{t, \tau}(x)\right)}{\sigma(t-\tau)} \int_{\tau}^{t} \eta^{x}(\theta) d \hat{w}(\theta) d \tau\right] .
$$

Note that to derive the second term in the right hand side of (3.9) we need a specific integration by parts formula called the Bismut-Elworthy-Li formula [12].

Since the system (3.3)- (3.8) is a closed system with respect to $\left(\psi_{t, 0}(x), \eta^{x}(t), u(t, x), p(t, x), \nabla u(t, x)\right)$, we aim to prove the existence and uniqueness theorem for its solution. At the end we check that the functions $(u(t, x), p(t, x))$ given by (3.4)- (3.5) satisfy (3.1), $(3.2)$.

To construct the solution of (3.4) - (3.8) we consider a system of successive approximations and prove their convergence.

Set

$$
u^{1}(t, x)=u_{0}(x), \quad \psi_{t, 0}^{0}(x)=x, \quad p^{1}(t, x)=0
$$

and consider stochastic processes $\psi_{t, \theta}^{k}(x)$, vector fields $u^{k}(t, x)$ and scalar functions $p^{k}(t, x)$ given by the following relations

$$
\begin{gathered}
d \psi_{t, \theta}^{k}=-u^{k}\left(\theta, \psi_{t, \theta}^{k}\right) d \theta+\sigma d \hat{w}(\theta), \quad \psi_{t, t}^{k}=x, \\
u^{k+1}(t, x)=E\left[u_{0}\left(\psi_{t, 0}^{k}(x)\right)-\int_{0}^{t} \nabla p^{k+1}\left(\tau, \psi_{t, \tau}^{k}(x)\right) d \tau\right], \\
-2 p^{k+1}(t, x)=\int_{0}^{\infty} E\left[\gamma^{k}(t, x+B(\tau))\right] d \tau
\end{gathered}
$$

where

$$
\left.\gamma^{k}(t, x)=\operatorname{Tr}\left[\nabla u^{k}\right]^{2}(t, x)\right]
$$

Finally, we consider $\eta_{t, \theta}^{x, k}, \nabla u^{k+1}(t, x)$ and $\nabla p^{k+1}(t, x)$ defined respectively by

$$
d \eta_{t, \theta}^{x, k}=-\nabla u^{k}\left(\theta, \psi_{t, \theta}^{k}\right) \eta_{t, \theta}^{x, k} d \theta, \quad \eta_{t, t}^{x, k}=I,
$$

and

$$
\begin{gathered}
\nabla u^{k+1}(t, x)=E\left[\nabla u_{0}\left(\psi_{t, 0}^{k+1}(x)\right) \eta_{t, 0}^{x, k}-\int_{0}^{t} \nabla^{2} p^{k+1}\left(\tau, \psi_{t, \tau}^{k}(x)\right) \eta_{t, \tau}^{x, k} d \tau\right] \\
-2 \nabla p^{k+1}(t, x)=\int_{0}^{\infty} \frac{1}{\tau} E\left[\gamma^{k}(t, x+B(\tau)) B(\tau)\right] d \tau
\end{gathered}
$$

Note that for $k=1$ we have

$$
d \psi_{t, \theta}^{1}=-u_{0}\left(\theta, \psi_{t, \theta}^{1}\right) d \theta+\sigma d \hat{w}(\theta), \quad \psi_{t, t}^{1}=x
$$


that is we can solve the stochastic equation (3.4) independently on (3.5)-(3.6). Then given the process $\psi_{t, 0}^{1}(x)$ and keeping in mind the properties of the function $p^{1}$ that satisfies the Poisson equation

$$
-\Delta p^{1}(t, x)=\gamma^{0}(t, x),
$$

we compute $u^{1}(t, x)$ from (3.12). Next we compute $\nabla u^{1}(t, x), \nabla p^{1}(t, x)$ from (3.16), (3.17) and proceed to $k=2$.

To prove the convergence of the successive approximations obtained in this way we need to derive some apriori estimates.

Let $g(t, x) \in R^{3}$ be a given bounded Lipschitz continuous function on $[0, \infty) \times R^{3}$. Set $g(t, \psi(t, x))=$ $g(t) \circ \psi(t)(x)$ for any functions $\left.\psi(t, x) \in R^{3}\right)$.

Consider the stochastic equation

$$
d \psi_{t, \theta}^{g}=-g(\theta) \circ \psi_{t, \theta}^{g} d \theta+\sigma d \hat{w}(\theta), \quad \psi_{t, t}^{g}(x)=x
$$

and define the vector fields $u^{g}(t, x)$ and $\nabla p^{g}(t, x)$ by

$$
\begin{gathered}
u^{g}(t, x)=E\left[u_{0}\left(\psi_{t, 0}^{g}(x)\right)-\int_{0}^{t} \nabla p^{g}\left(\tau, \psi_{t, \tau}^{g}(x)\right) d \tau\right], \\
-2 p^{g}(t, x)=\int_{0}^{\infty} E\left[\gamma^{g}(t, x+B(\tau))\right] d \tau,
\end{gathered}
$$

where

$$
\gamma^{g}(t, x)=\operatorname{Tr}[\nabla g]^{2}(t, x) .
$$

We derive formally from (3.21) by the integration by parts formula (Bismut - Elworthy - Li formula [12]) that

$$
-2 \nabla p^{g}(t, x)=\int_{0}^{\infty} E\left[\frac{1}{\tau} \gamma^{g}(t, x+B(\tau)) B(\tau)\right] d \tau .
$$

Below we will describe the conditions on $\gamma$ which justify (3.23).

Condition C 3.1 Let $g(t, x) \in R^{3}$ be a divergent free vector field depending on time and defined on $[0, T] \times R^{3}$ for a certain constant $T>0$. We assume that $g(t)$ belongs to $\mathbf{C}^{1, \alpha}\left(R^{3}\right), 0<\alpha \leq 1$ for a fixed $t \in[0, T]$ and satisfies the following estimates:

1. $\|g(t)\|_{q, l o c} \leq N_{g}(t)$ for some $q$ to be specified below, $\|g(t)\|_{\infty} \leq K_{g}(t)$ and

$$
\|g(t, x)-g(t, y)\| \leq L_{g}(t)\|x-y\|, \quad\|\nabla g(t, x)-\nabla g(t, y)\| \leq L_{g}^{1}(t)\|x-y\| .
$$

2. $\|\nabla g(t)\|_{\infty} \leq K_{g}^{1}(t), \quad\|\nabla g(t)\|_{r, l o c} \leq N_{g}^{1}(t)$.

Here $K_{g}(t), L_{g}(t), N_{g}(t)$ and $K_{g}^{1}(t), L_{g}^{1}(t), N_{g}^{1}(t)$ are positive continuous functions defined on an interval $[0, T]$ with $T>0, r=m$ and $r=q$ for $1<q<\frac{3}{2}<3<m<\infty$.

Set $\psi^{g}(\tau)=\psi_{t, \tau}^{g}(x)$ and consider the stochastic equation

$$
\psi^{g}(\tau)=x-\int_{\tau}^{t} g\left(\tau_{1}, \psi^{g}\left(\tau_{1}\right)\right) d \tau_{1}+\int_{\tau}^{t} \sigma d \hat{w}\left(\tau_{1}\right)
$$


with $0 \leq \tau \leq t<T$. When we are interested in the particular dependence of the process $\psi^{g}(\tau)$ on the parameters $t, x$ we write $\psi^{g}(\tau)=\psi_{t, x}^{g}(\tau)$ or $\psi^{g}(\tau)=\psi_{t, \tau}^{g}(x)$.

Lemma 3.1 Assume that C 3.1 holds. Then there exists a unique solution $\psi^{g}(\tau)$ of $(3.24)$ and the following estimates

$$
\begin{gathered}
E\left\|\psi^{g}(\tau)\right\|^{2} \leq 3\left[\|x\|^{2}+\sigma^{2}(t-\tau)+(t-\tau) \int_{\tau}^{t}\left[K_{g}^{2}\left(\tau_{1}\right)\right] d \tau_{1}\right] \\
E\left\|\psi_{t, x}^{g}(\tau)-\psi_{t, y}^{g}(\tau)\right\| \leq\|x-y\| e^{\int_{\tau}^{t} L_{g}(\theta) d \theta} \\
E\left\|\psi^{g}(\tau)-\psi^{g_{1}}(\tau)\right\| \leq \int_{\tau}^{t}\left\|g\left(\tau_{1}\right)-g_{1}\left(\tau_{1}\right)\right\|_{\infty} d \tau_{1} e^{\int_{\tau}^{t} L_{g}(\theta) d \theta}
\end{gathered}
$$

hold.

Proof. The proof of the estimates of this lemma is standard and based on estimates of classical and stochastic integrals. We only show the proof of (3.26). In view of $\mathbf{C} \mathbf{3 . 1}$ we have

$$
E\left\|\psi_{t, x}^{g}(\tau)-\psi_{t, y}^{g}(\tau)\right\| \leq\|x-y\|+\int_{\tau}^{t} L_{g}\left(\tau_{1}\right)\left\|\psi_{t, x}^{g}\left(\tau_{1}\right)-\psi_{t, y}^{g}\left(\tau_{1}\right)\right\| d \tau_{1}
$$

where $0 \leq \tau \leq t \leq T$ with some constant $T$ to be chosen later. Finally, by Gronwall's lemma, we get

$$
E\left\|\psi_{t, x}^{g}(\tau)-\psi_{t, y}^{g}(\tau)\right\| \leq\|x-y\| e^{\int_{\tau}^{t} L_{g}(\theta) d \theta}
$$

Along with (3.19)-(3.22) we need the equations for the mean square derivative $\eta^{g}(t)=\nabla \psi_{t, 0}^{g}(x)$ of the diffusion process $\psi_{t, 0}^{g}(x)$ that satisfies (3.19), and the gradient $v(t, x)=\nabla u^{g}(t, x)$ of the function $u^{g}(t, x)$ given by $(3.20)$.

Lemma 3.2 Assume that $\mathbf{C} \mathbf{3 . 1}$ holds. Then the process $\eta^{x, g}(\tau)=\nabla \psi_{t, \tau}^{g}(x)$ satisfies the stochastic equation

$$
d \eta^{x, g}(\tau)=-\nabla g\left(\tau, \psi_{t, \tau}^{x, g}(x)\right) \eta^{x, g}(\tau) d \tau, \quad \eta^{x, g}(t)=I .
$$

The process $\eta^{x, g}(\tau)$ possesses the following properties.

The determinant $\operatorname{det} \eta^{\mathrm{g}}(\tau)$ is equal to 1 , i. e. $\operatorname{det} \eta^{g}(\tau)=J_{t, \tau}=1$ and

$$
\begin{gathered}
E\left\|\eta^{x, g}(\tau)\right\| \leq e^{\int_{\tau}^{t} K_{g}^{1}(\theta) d \theta} \\
E\left\|\eta^{x, g}(\tau)-\eta^{y, g}(\tau)\right\| \leq C\|x-y\|
\end{gathered}
$$

with some positive constant $C$ depending on $t, \tau$ and $g$.

In addition the following integration by part formula is valid

$$
\int_{R^{3}} f\left(\psi_{t, x}^{g}(\tau)\right) d x=\int_{R^{3}} f(x) d x, \quad f \in L^{1}\left(R^{3}\right) .
$$

Proof. Under C 3.1 the first statement immediately follows from general results of the stochastic differential equation theory. By direct computation one can check that $J_{t, \tau}$ satisfies the linear equation

$$
d J_{t, \tau}=-\operatorname{div} g\left(\psi_{t, \tau}^{g}\right) J_{t, \tau} d \tau, \quad J_{t, t}=I
$$


and since div $g=0$ we get the second statement. Besides $\psi_{t, \tau}^{g}$ is a $C^{1}$ stochastic diffeomorphism (see [5]) and hence the integration by part formula (3.31) holds. Finally (3.29) is deduced from the inequality

$$
E\left\|\eta^{x, g}(\tau)\right\| \leq 1+\int_{\tau}^{t} K_{g}^{1}(\theta) E\left\|\eta^{x, g}(\theta)\right\| d \theta
$$

by the Gronwall lemma.

One can easily check that for the solution $\eta^{x, g}(t)$ of (3.28) we have

$$
\begin{gathered}
E\left\|\eta^{x, g}(\tau)-\eta^{y, g}(\tau)\right\| \leq \int_{\tau}^{t} E\left\|\nabla g\left(\theta, \psi_{t, \theta}^{g}(x)\right)-\nabla g\left(\theta, \psi_{t, \theta}^{g}(y)\right)\right\| d \theta e^{\int_{\tau}^{t} K_{g}^{1}(\theta) d \theta} \leq \\
\left.\left.E \int_{\tau}^{t} L_{g}^{1}(\theta) E \| \psi_{t, \theta}^{g}(x)\right)-\psi_{t, \theta}^{g}(y)\right) \| d \theta
\end{gathered}
$$

and by (3.26) we derive (3.30).

Let us state conditions on the initial data $u_{0}$ of the N-S system.

We say that $\mathbf{C} \mathbf{3 . 2}$ holds when

i) for some $0<\alpha \leq 1$ the initial vector field $u_{0} \in C_{0}^{1+\alpha}\left(R^{3}\right)$ satisfies the estimates

$$
\left\|u_{0}\right\|_{\infty} \leq K_{0}, \quad\left\|\nabla u_{0}\right\|_{\infty} \leq K_{0}^{1}, \quad\left\|u_{0}\right\|_{r, l o c} \leq M_{0}, \quad\left\|\nabla u_{0}\right\|_{r, l o c} \leq M_{0}^{1}
$$

with some positive constants $K_{0}, K_{0}^{1}, M_{0}, M_{0}^{1}$ and $r$.

ii) $u_{0}$ and $\nabla u_{0}$ are Lipschitz-continuous with positive Lipschitz constants $L_{0}$ and $L_{0}^{1}$ respectively.

Keeping in mind conditions $\mathbf{C} \mathbf{3 . 1}$ and $\mathbf{C} \mathbf{3 . 2}$ we derive estimates for $u^{g}(t)$ defined by (3.20) on a certain time interval $[0, T]$ and its gradient $\nabla u^{g}(t, x)$.

Lemma 3.3 Assume that $g(t, x)$ satisfies $\mathbf{C} \mathbf{3 . 1}$ and $u_{0}$ satisfies $\mathbf{C} \mathbf{3 . 2}$ with $r=q$ and $r=m$ for $1<q<\frac{3}{2}<3<m<\infty$. Then the vector field $u^{g}(t, x)$ given by (3.20) satisfies the estimate

$$
\left\|u^{g}(t)\right\|_{\infty} \leq K_{0}+\int_{0}^{t} C_{q m} K_{g}^{1}(\tau)\left[\|\nabla g(\tau)\|_{q, l o c}+\|\nabla g(\tau)\|_{m, l o c}\right] d \tau
$$

Under the conditions of this lemma the proof of (3.32) can be easily obtained by a direct computation from (3.20) using the estimates of the Newton potential given in lemma 3.4 below.

Lemma 3.4 Let $G \subset R^{3}$ be a bounded domain and $\gamma^{g} \in L^{q}(G) \cap L^{m}(G)$ for some $1 \leq q<\frac{3}{2}<$ $3<m<\infty$ and

$$
-\Delta p(t, x)=\gamma^{g}(t, x), \quad x \in G .
$$

Then $\left\|\nabla p^{g}\right\|_{\infty} \leq C_{q m}\left(\left\|\gamma^{g}\right\|_{q, l o c}+\left\|\gamma^{g}\right\|_{m, l o c}\right)$ and

$$
\left\|\nabla_{i} \nabla_{j} p^{g}\right\|_{\infty} \leq C\left(\left\|\gamma^{g}\right\|_{q, l o c}+\left[\gamma^{g}\right]_{\alpha}\right) .
$$

3. Let $\gamma^{g} \in L^{r}(G)$ for $1<r<\infty$. Then $p^{g} \in W^{2, r}\left(R^{3}\right)$ and the Calderon- Zygmund inequality holds

$$
\left\|\nabla^{2} p^{g}\right\|_{r, l o c} \leq C_{1}\left\|\gamma^{g}\right\|_{r, l o c}
$$


The proof of these estimates for a solution of the Poisson equation can be found in the book by Gilbarg and Trudinger ([13] Th 9.9). The probabilistic proof of some of these estimates can be found in $[4]$.

Lemma 3.5 Assume that the conditions of lemma 3.3 hold and $u^{g}(t, x)$ is given by (3.20). Then the function $\nabla u^{g}(t, x)$ admits a representation of the form

$$
\nabla u^{g}(t, x)=E\left[\nabla u_{0}\left(\psi_{t, 0}^{g}(x)\right) \eta^{x, g}(t)-\int_{0}^{t} \nabla^{2} p^{g}\left(\tau, \psi_{t, \tau}^{g}(x)\right) \eta^{x, g}(\tau) d \tau\right]
$$

and the estimate

$$
\begin{gathered}
\left\|\nabla u^{g}(t)\right\|_{\infty} \leq e^{\int_{0}^{t} K_{g}^{1}(\theta) d \theta} K_{0}^{1}+ \\
\int_{0}^{t} e^{\int_{\tau}^{t} K_{g}^{1}(\theta) d \theta} K_{g}^{1}(\tau)\left[\|\nabla g(\tau)\|_{q, l o c}+\|\nabla g(\tau)\|_{m, l o c}\right] d \tau
\end{gathered}
$$

holds for $1<q<\frac{3}{2}<3<m<\infty, 0 \leq t \leq T$.

Proof. The formal differentiation of (3.20) in $x$ justified by $\mathbf{C ~ 3 . 1 , ~ C ~ 3 . 2 ~ a n d ~ t h e ~ r e s u l t s ~ o f ~ l e m m a ~}$ 3.4 yields (3.33). To verify the estimate (3.34) we use the above estimates for the process $\eta^{x, g}(t)$ and the estimates of the Newton potential derivative from lemma 3.4. Hence we obtain

$$
\begin{gathered}
\left\|\nabla u^{g}(t)\right\|_{\infty} \leq K_{0}^{1} \int_{0}^{t} K_{g}^{1}(\theta) d \theta+ \\
\left.\int_{0}^{t} C_{q m} e^{\int_{\tau}^{t} K_{g}^{1}(\theta) d \theta}\left[\left\|\operatorname{Tr}[\nabla g(\tau)]^{2}\right\|_{m, l o c}+\left\|\operatorname{Tr}[\nabla g(\tau)]^{2}\right\|_{q, l o c}\right] d \tau\right]
\end{gathered}
$$

that immediately leads to (3.34).

Now we have to derive the estimate for the function $\|\nabla u(t)\|_{r, l o c}$.

Lemma 3.6 Assume that the conditions of lemma 3.3 hold. Then for $1<r<\infty$ the gradient of the function $u^{g}(t, x)$ given by (3.20) satisfies the estimate

$$
\left\|\nabla u^{g}(t)\right\|_{r, l o c} \leq e^{2 \int_{0}^{t} K_{g}^{1}(\theta) d \theta}\left[\left\|\nabla u_{0}\right\|_{r, l o c}+C \int_{0}^{t}\|\nabla g(\tau)\|_{r, l o c} d \tau\right],
$$

where $0 \leq t \leq T$ and $C$ depends on $r$ and $T$.

Proof. Let us derive the $L^{p}$ - estimate for $\nabla u^{g}(t, x)$ given by (3.33). To derive the estimate for $\left\|\nabla u^{g}(t)\right\|_{r, l o c}^{r}=\int_{K}\left\|\nabla u^{g}(t, x)\right\|^{r} d x$ (where $K$ is an arbitrary compact in $G$ ) we apply first the triangle inequality to obtain

$$
\left\|\nabla u^{g}(t)\right\|_{r, l o c} \leq \alpha_{1}+\alpha_{2},
$$

where

$$
\begin{gathered}
\alpha_{1}=\left(\int_{K} E\left[\left\|\nabla u_{0}\left(\psi_{t, 0}^{g}(x)\right) \eta^{x, g}(t)\right\|^{r}\right] d x\right)^{\frac{1}{r}}, \\
\left.\alpha_{2}=\left(\int_{K} \int_{0}^{t} E \| \nabla^{2} p^{g}\left(\tau, \psi_{t, \tau}^{g}(x)\right)\right) \eta^{x, g}(\tau) \|^{r} d \tau d x\right)^{\frac{1}{r}} .
\end{gathered}
$$

To estimate $\alpha_{1}$ we apply the Hölder inequality and take into account the inequality (3.29) for the process $\eta^{x, g}(\tau)$. Besides we recall that $\psi_{t, \tau}(x)$ preserves the volume. As a result we have

$$
\left.\alpha_{1} \leq\left(\int_{K}\left(E\left[\left\|\nabla u_{0}\left(\psi_{t, 0}^{g}(x)\right)\right\|^{2}\right] E\left[\left\|\eta^{x, g}(t)\right\|^{2}\right]\right)^{\frac{r}{2}} d x\right)\right)^{\frac{1}{r}} \leq\left\|\nabla u_{0}\right\|_{r, l o c} e^{\int_{0}^{t} K_{g}^{1}(\theta) d \theta} .
$$


To derive the estimate for $\alpha_{2}$ we deduce from the Calderon-Zygmund inequality (see lemma 3.4) and the estimate of $\eta^{x, g}(t)$ that

$$
\alpha_{2}^{r} \leq C_{r} \int_{0}^{t} e^{\int_{0}^{\tau} K_{g}^{1}(\theta) d \theta} K_{g}^{1}(\tau) \int_{K}\|\nabla g(\tau, x)\|^{r} d x d \tau .
$$

Combining the above estimates for $\alpha_{1}$ and $\alpha_{2}$ we obtain the required estimate

$$
\begin{aligned}
& \left\|\nabla u^{g}(t)\right\|_{r, l o c} \leq e^{\int_{0}^{t} K_{g}^{1}(\theta) d \theta}\left[\left\|\nabla u_{0}\right\|_{r, l o c}+\right. \\
& \left.C_{r} \int_{0}^{t} e^{\int_{0}^{\tau} K_{g}^{1}(\theta) d \theta} K_{g}^{1}(\tau)\|\nabla g(\tau)\|_{r, l o c} d \tau\right]
\end{aligned}
$$

Finally we get

$$
\left\|\nabla u^{g}(t)\right\|_{r, l o c} \leq e^{2 \int_{0}^{t} K_{g}^{1}(\theta) d \theta}\left[\left\|\nabla u_{0}\right\|_{r, l o c}+C \int_{0}^{t}\|\nabla g(\tau)\|_{r, l o c} d \tau\right],
$$

where $C$ depends on $r$ and $T$.

Theorem 3.7 Assume that conditions C 3.1 and C 3.2 hold. Then there exists an interval $\Delta_{1}=\left[0, T_{1}\right]$ and functions $\alpha(t), \beta(t), \kappa(t)$ bounded for $t \in \Delta_{1}$, such that, if for all $t \in \Delta_{1},\|g(t)\|_{\infty} \leq$ $\kappa(t)$ and $\|\nabla g(t)\|_{\infty} \leq \alpha(t),\|\nabla g(t)\|_{r, l o c} \leq \beta_{r}(t)$ then the function $\left\|\nabla u^{g}(t, x)\right\|$ (where $u^{g}(t, x)$ is given by (3.20)) satisfies the estimates

$$
\left\|u^{g}(t)\right\|_{\infty} \leq \kappa(t), \quad\left\|\nabla u^{g}(t)\right\|_{\infty}^{2} \leq \alpha(t), \quad\left\|\nabla u^{g}(t)\right\|_{r, l o c}^{2} \leq \beta_{r}(t)
$$

for $r=q$ and $r=m$ and $1<m<\frac{3}{2}<3<q<\infty$.

Proof. Analyzing the above estimates (3.35), (3.36) for the functions $u^{g}(t, x)$ and $\nabla u^{g}(t, x)$ we note that to prove the required estimates it is enough to construct the solutions of the following integral equations

$$
\begin{gathered}
\alpha(s)=e^{\int_{s}^{t} \alpha(\theta) d \theta} K_{0}^{1}+C_{q m} \int_{s}^{t} e^{\int_{s}^{\tau} \alpha(\theta) d \theta} \alpha(\tau)\left[n_{q}(\tau)+n_{m}(\tau)\right] d \tau, \\
n_{r}(s)=e^{\int_{s}^{t} \alpha(\theta) d \theta}\left\|\nabla u_{0}\right\|_{r}+C_{r} \int_{s}^{t} e^{\int_{s}^{\tau} \alpha(\theta) d \theta} n_{r}(\tau) \alpha(\tau) d \tau
\end{gathered}
$$

for $r=q$ and $r=m$ and $C_{q m}=\max \left(C_{q}, C_{m}\right)$ and

$$
\beta(s)=e^{\int_{s}^{t} \alpha(\theta) d \theta} \beta_{0}+C_{q m} \int_{s}^{t} e^{\int_{s}^{\tau} \alpha(\theta) d \theta} \alpha(\tau) \beta(\tau) d \tau,
$$

where $\beta(\tau)=n_{q}(\tau)+n_{m}(\tau)$, and

$$
\left\|\nabla u_{0}\right\|_{q, l o c}+\left\|\nabla u_{0}\right\|_{m, l o c}=n_{q}(0)+n_{m}(0)=\beta_{0} .
$$

To construct the solution of the above system of integral equations (3.40)-(3.42) we consider the system of ODEs

$$
\begin{gathered}
\frac{d \alpha}{d s}=-\alpha^{2}(s)-C_{q m} \alpha(s) \beta(s), \quad \alpha(t)=K_{0}^{1}, \\
\frac{d \beta}{d s}=-\alpha(s) \beta(s)-C_{q m} \alpha(s) \beta(s), \quad \beta(t)=\beta_{0} .
\end{gathered}
$$


By classical results of the ODE theory we know that there exists an interval $\left[0, T_{1}\right]$ depending on $K_{0}^{1}, N_{0}^{1}$ and $C, C_{q m}$ such that the system (3.41), (3.42) has a bounded solution defined on this interval.

To prove the convergence for $k \rightarrow \infty$ of functions $u^{k}(t, x), \nabla u^{k}(t, x)$ we need one more auxiliary estimate. Actually, we have proved that $u^{k}(t)$ is Lipschitz-continuous with the Lipschitz constant independent of $k$. It remains to prove that $\nabla u^{k}(t)$ has the same property.

Lemma 3.8 Assume that $\mathbf{C} 3.1$ and $\mathbf{C}$ 3.2 hold. Then the function $\nabla u^{g}(t, x)$ defined in lemma 3.5 admits a representation of the form

$$
\begin{gathered}
\nabla u^{g}(t, x)=E\left[\nabla u_{0}\left(\psi_{t, 0}^{g}(x)\right) \eta^{x, g}(t)-\right. \\
\left.\int_{0}^{t} \frac{1}{\sigma(t-\tau)} \nabla p^{g}\left(\tau, \psi_{t, \tau}^{g}(x)\right) \int_{\tau}^{t} \eta^{x, g}(\theta) d \hat{w}(\theta) d \tau\right]
\end{gathered}
$$

and satisfies the estimate

$$
\left\|\nabla u^{g}(t, x)-\nabla u^{g}(t, y)\right\| \leq N_{1}^{g}(t)\|x-y\| \text { if } t \in\left[0, T_{1}\right]
$$

for any $x, y \in G$ where $G$ is a compact in $R^{3}$ and the positive function $N_{1}^{g}(t)$ depending on the parameters in conditions $\mathbf{C} \mathbf{3 . 1}$ and $\mathbf{C} \mathbf{3 . 2}$ is bounded over the interval $\left[0, T_{1}\right]$ defined in theorem 3.7 .

Proof. To derive (3.43) we compute directly the gradient of the first term in (3.20) and apply the Bismut-Elworthy -Li formula [12] to compute the gradient of the second term in this relation. Next we use the representation (3.43) to deduce the Lipschitz estimate for the gradient of the function $u(t, x)$ . As a result we have

$$
\left\|\nabla u^{g}(t, x)-\nabla u^{g}(t, y)\right\| \leq \kappa_{1}+\kappa_{2}+\kappa_{3}+\kappa_{4},
$$

where

$$
\begin{gathered}
\kappa_{1}(t)=E\left[\left\|\nabla u_{0}\left(\psi_{t, 0}^{g}(x)\right)-\nabla u_{0}\left(\psi_{t, 0}^{g}(y)\right)\right\|\left\|\eta^{x, g}(t)\right\|\right], \\
\kappa_{2}(t)=E\left[\left\|\nabla u_{0}\left(\psi_{t, 0}^{g}(y)\right)\right\|\left\|\eta^{x, g}(0)-\eta^{y, g}(0)\right\|\right], \\
\kappa_{3}(t)=\int_{0}^{t} E\left[\frac{\left\|\nabla p^{g}\left(\tau, \psi_{t, \tau}^{g}(x)\right)-\nabla p^{g}\left(\tau, \psi_{t, \tau}^{g}(y)\right)\right\|}{\sigma(t-\tau)}\left\|\int_{\tau}^{t} \eta^{x, g}(\theta) d \hat{w}(\theta)\right\|\right] d \tau, \\
\kappa_{4}(t)=\int_{0}^{t} \frac{1}{\sigma(t-\tau)} E\left[\left\|\nabla p^{g}\left(\tau, \psi_{t, \tau}^{g}(y)\right)\right\| \int_{\tau}^{t}\left[\eta^{x, g}(\theta)-\eta^{y, g}(\theta)\right] d \hat{w}(\theta) \|\right] d \tau .
\end{gathered}
$$

One can easily check using the estimates stated in lemmas $3.3-3.5$ that under the conditions $\mathbf{C}$

\section{1, C 3.2}

$$
\begin{gathered}
\kappa_{1}(t) \leq L_{0}^{1} E\left\|\psi_{t, 0}^{g}(x)-\psi_{t, 0}^{g}(y)\right\| e^{\int_{0}^{t} K_{g}^{1}(\theta) d \theta} \leq \\
\|x-y\| L_{0}^{1} e^{\int_{\tau}^{t}\left[L_{g}(\theta)+K_{g}^{1}(\theta)\right] d \theta}=\Theta_{1}\|x-y\|
\end{gathered}
$$

and

$$
\kappa_{2}(t) \leq K_{0}^{1} E\left\|\eta^{x, g}(t)-\eta^{y, g}(t)\right\| \leq\|x-y\| \int_{\tau}^{t} K_{g}^{1}(\theta) e^{\int_{\theta}^{t} K_{g}^{1}\left(\theta_{1}\right) d \theta_{1}} d \theta=\Theta_{2}\|x-y\|
$$


To derive the estimates for $\kappa_{3}$ and $\kappa_{4}$ we apply the inequalities $\left\|\nabla_{i} \nabla_{j} p^{g}\right\|_{\infty} \leq C\left(\left\|\gamma^{g}\right\|_{q, l o c}+\right.$ $\left.\left[\gamma^{g}\right]_{1, G}\right),\left\|\nabla_{i} \nabla_{j} p^{g}\right\|_{r, l o c} \leq\left\|\gamma^{g}\right\|_{r, l o c}$ from lemma 3.4. This yields

$$
\begin{gathered}
\kappa_{3}(t) \leq \int_{0}^{t} E\left[\left\|\nabla^{2} p^{g}(\tau)\right\|_{\infty} \frac{\left\|\psi_{t, 0}^{g}(x)-\psi_{t, 0}^{g}(y)\right\|}{\sigma \sqrt{t-\tau}}\left\|\eta^{x, g}(\tau)\right\|\right] d \tau \leq \\
\int_{0}^{t} \frac{\left(E\left\|\psi_{t, \tau}^{g}(x)-\psi_{t, \tau}(y)\right\|^{2}\right)^{\frac{1}{2}}}{\sigma \sqrt{t-\tau}}\left(\left\|\gamma^{g}(\tau)\right\|_{q, l o c}+\left[\gamma^{g}(\tau)\right]_{1, G}\right) e^{\int_{\tau}^{t} K_{g}^{1}(\theta) d \theta} d \tau \leq \\
\Theta_{1}\|x-y\| \int_{0}^{t} \frac{\left\|\gamma^{g}(\tau)\right\|_{q, l o c}+\left[\gamma^{g}(\tau)\right]_{1, G}}{\sigma \sqrt{t-\tau}} e^{\int_{\tau}^{t}} K_{g}^{1}(\theta) d \theta d \tau \leq \\
\Theta_{3} \Theta_{1}\|x-y\| \sigma^{-1}\left(\sup _{0 \leq \tau \leq t}[\beta(\tau)] \sqrt{t}+\int_{0}^{t} \frac{s(\tau)}{\sqrt{t-\tau}} d \tau\right)
\end{gathered}
$$

and

$$
\begin{gathered}
\kappa_{4}(t) \leq \int_{0}^{t} \frac{C_{q m}\left(\left\|\gamma^{g}(\tau)\right\|_{q, l o c}+\left\|\gamma^{g}(\tau)\right\|_{m, l o c}\right)}{\sigma \sqrt{t-\tau}}\left(E\left\|\eta^{x, g}(\tau)-\eta^{y, g}(\tau)\right\|^{2}\right)^{\frac{1}{2}} d \tau \leq \\
2 \sup _{0 \leq \tau \leq t} \beta(\tau)\|x-y\| \int_{0}^{t} \frac{\Theta_{2} C_{q m}}{\sigma \sqrt{t-\tau}} d \tau=2 \Theta_{4}\|x-y\| \frac{\Theta_{2} C_{q m}}{\sigma} \sqrt{t} .
\end{gathered}
$$

Here $\Theta_{3}=e^{\int_{0}^{t} K_{g}^{1}(\tau) d \tau}, s(\tau)=\left[\gamma^{g}(\tau)\right]_{1, G}$ and $\beta(\tau)$ is defined in theorem 3.7. Finally, combining the above estimates for $\kappa_{i}, i=1,2,3,4$, we obtain

$$
s(t) \leq \Theta_{5}+\Theta_{6} \int_{0}^{t} \frac{s(\tau)}{\sqrt{t-\tau}} d \tau
$$

and applying the Gronwall lemma we derive the estimate

$$
s(t) \leq \Theta_{5} e^{\Theta_{6} \sqrt{t}}
$$

where $\Theta_{i}, i=5,6$ depend on the parameters in conditions $\mathbf{C ~} 3.1$ and $\mathbf{C ~ 3 . 2 , \sigma}$ and $T_{1}$ for $0 \leq t \leq T_{1}$, where $T_{1}$ is defined in theorem 3.7 .

The estimates of theorem 3.7 and lemma 3.8 allow to prove the uniform convergence on compacts of the successive approximations (3.10)-(3.14) for the solutions of the system (3.4) - (3.6) in $C\left(\left[0, T_{1}\right]\right.$, $\left.C^{1, \alpha}(K)\right) \cap C\left(\left[0, T_{1}\right], L^{m}(K) \cap L^{q}(K)\right)$ for $1<q<\frac{3}{2}<3<m<\infty$ and arbitrary compact $K$ in $G$. In particular, they justify the possibility to differentiate the system (3.10)-(3.14) in $x$ for each $k$ and to consider the following equations

$$
d \eta_{t, \theta}^{k, x}=-\nabla u^{k}\left(\theta, \psi_{t, \theta}^{k}\right) \eta_{t, \theta}^{x, k} d \theta, \quad \eta_{t, t}^{x, k}=I
$$

where $I$ is the identity matrix acting in $R^{3}$ and

$$
\begin{gathered}
\nabla u^{k+1}(t, x)=E\left[\nabla u_{0}\left(\psi_{t, 0}^{k+1}(x)\right) \eta_{t, 0}^{x, k}-\right. \\
\left.\int_{0}^{t} \frac{1}{\sigma(t-\tau)} \nabla p^{k+1}\left(\tau, \psi_{t, \tau}^{k}(x)\right) \int_{\tau}^{t} \eta_{t, \theta}^{x, k} d \hat{w}(\theta) d \tau\right], \\
-2 \nabla p^{k+1}(t, x)=\int_{0}^{\infty} \frac{1}{\tau} E\left[\gamma^{k}(t, x+B(\tau)) B(\tau)\right] d \tau,
\end{gathered}
$$

where $\gamma^{k}=\operatorname{Tr}\left[\nabla u^{k}\right]^{2}$. 
Now we can prove the following assertion.

Theorem 3.9 Assume that $\mathbf{C}$ 3.2 holds. Then if $k \rightarrow \infty$ the functions $u^{k}(t), \nabla u^{k}(t, x)$ determined by (3.11) and (3.45) uniformly converge on compacts to limiting functions $u(t), \nabla u(t)$ satisfying (3.4) and (3.8)and $u(t) \in \mathbf{C}\left(\left[0, T_{1}\right], \mathbf{C}^{1, \alpha}\right), \nabla u(t) \in \mathbf{C}\left(\left[0, T_{1}\right], \mathbf{C}^{0, \alpha}\right), 0<\alpha \leq 1$ for all $t \in\left[0, T_{1}\right]$. Here $\left[0, T_{1}\right]$ is the interval where the solution $(\alpha(t), \beta(t))$ of the system (3.41), (3.42) is bounded. In addition the estimates $\|\nabla u(t)\|_{\infty} \leq \alpha(t),\|\nabla u(t)\|_{q, l o c} \leq \beta(t)$ hold for $1<q<\frac{3}{2}, t \in\left[0, T_{1}\right]$.

Proof. By theorem 3.7 we know that the mapping

$$
\Phi(t, x, g)=E\left[u_{0}\left(\psi_{t, 0}^{g}(x)\right)-\int_{0}^{t} \nabla p^{g}\left(\tau, \psi_{t, \tau}^{g}(x)\right) d \tau\right]
$$

acts in the space $\mathbf{C}^{1, \alpha} \cap \mathbf{L}_{q, \text { loc }} \cap \mathbf{L}_{m, l o c}$ (for a fixed $t \in\left[0 . T_{1}\right]$ ) with $1<q<\frac{3}{2}<3<m<\infty$.

Consider the successive approximations (3.10) -(3.14) and (3.44) - (3.46), set

$$
\begin{gathered}
S^{k+1}(t, x)=\left\|u^{k+1}(t, x)-u^{k}(t, x)\right\|, \\
n^{k+1}(t, x)=\left\|\nabla u^{k+1}(t, x)-\nabla u^{k}(t, x)\right\|
\end{gathered}
$$

and

$$
\begin{aligned}
& l^{k}(t)=\left\|S^{k}(t)\right\|_{\infty}, \quad m_{r}^{k}(t)=\left\|S^{k}(t)\right\|_{r, l o c}, \\
& \rho^{k}(t)=\left\|n^{k}(t)\right\|_{\infty}, \quad \zeta_{r}^{k}(t)=\left\|n^{k}(t)\right\|_{r, l o c} .
\end{aligned}
$$

Then we obtain

$$
\begin{gathered}
n^{k+1}(t, x) \leq L_{0}^{1}\left(E\left[\left\|\psi_{t, 0}^{k}(x)-\psi_{t, 0}^{k-1}(x)\right\|\left\|\eta_{t, 0}^{x, k}\right\|\right]+\right. \\
\left.E\left[\left\|\psi_{t, 0}^{k}(x)\right\|\left\|\eta_{t, 0}^{x, k}-\eta_{t, 0}^{x, k-1}\right\|\right]\right)+\int_{0}^{t} \frac{1}{\sigma(t-\tau)} E\left[\| \nabla p^{k+1}\left(\tau, \psi_{t, \tau}^{k}(x)\right)-\right. \\
\left.\nabla p^{k}\left(\tau, \psi_{t, \tau}^{k-1}(x)\right)\|\| \int_{\tau}^{t} \eta_{t, \theta}^{x, k} d \hat{w}(\theta) \|\right] d \tau+ \\
\int_{0}^{t} \frac{1}{\sigma(t-\tau)} E\left[\left\|\nabla p^{k}\left(\tau, \psi_{t, \tau}^{k}(x)\right)\right\| \int_{\tau}^{t}\left[\eta_{t, \theta}^{x, k}-\eta_{t, \theta}^{x, k-1}\right] d \hat{w}(\theta) \|\right] d \tau
\end{gathered}
$$

Recall that by lemmas $3.2,3.3$ we know that

$$
\begin{aligned}
& \sup _{x} E\left\|\psi_{t, 0}^{k}(x)-\psi_{t, 0}^{k-1}(x)\right\| \leq \int_{0}^{t}\left[\left\|u^{k}(\tau)-u^{k-1}(\tau)\right\|_{\infty}\right] d \tau e^{\int_{0}^{t} \alpha(\tau) d \tau}, \\
& \quad \sup _{x} E\left\|\eta_{t, 0}^{x, k}-\eta_{t, 0}^{x, k-1}\right\| \leq \int_{0}^{t}\left\|\nabla u^{k}(\tau)-\nabla u^{k-1}(\tau)\right\|_{\infty} d \tau e^{\int_{0}^{t} \alpha(\tau) d \tau} \\
& +\sup _{x} \int_{0}^{t} E\left\|\nabla u^{k-1}\left(\tau, \psi_{t, \tau}^{k}(x)\right)-\nabla u^{k-1}\left(\tau, \psi_{t, \tau}^{k-1}(x)\right)\right\| d \tau e^{\int_{0}^{t} \alpha(\tau) d \tau}
\end{aligned}
$$

and applying the estimates from theorem 3.7 we get

$$
\begin{aligned}
& \rho^{k+1}(t) \leq e^{\int_{0}^{t} \alpha(\tau) d \tau}\left[L_{0}^{1} \int_{0}^{t} \sup _{x} E\left\|u^{k}\left(\tau, \psi_{t, \tau}^{k}(x)\right)-u^{k-1}\left(\tau, \psi_{t, \tau}^{k-1}(x)\right)\right\| d \tau\right. \\
& \left.+\int_{0}^{t} \rho^{k}(\tau) d \tau+\sup _{x} \int_{0}^{t} E\left\|\nabla u^{k-1}\left(\tau, \psi_{t, \tau}^{k}(x)\right)-\nabla u^{k-1}\left(\tau, \psi_{t, \tau}^{k-1}(x)\right)\right\| d \tau\right]+
\end{aligned}
$$




$$
\begin{gathered}
\int_{0}^{t} \frac{C\left[\left\|\nabla u^{k}(\tau) \nabla u^{k}(\tau)\right\|_{q}+\left\|\nabla u^{k}(\tau) \nabla u^{k}(\tau)\right\|_{m}\right]\left(E\left\|\eta^{k}(\tau)-\eta^{k-1}(\tau)\right\|_{\infty}^{2}\right)^{\frac{1}{2}}}{\sigma \sqrt{t-\tau}} d \tau+ \\
\left.\int_{0}^{t} \frac{1}{\sigma \sqrt{t-\tau}} \sup _{x} E\left\|\nabla p^{k+1}\left(\tau, \psi_{t, \tau}^{k}(x)\right)-\nabla p^{k}\left(\tau, \psi_{t, \tau}^{k-1}(x)\right)\right\|^{2}\right)^{\frac{1}{2}} e^{\int_{\tau}^{t} \alpha(\theta) d \theta} d \tau .
\end{gathered}
$$

To derive the estimate for the last term of the above inequality we recall (see lemma 3.1 and lemma 3.4) that for $1<q<\frac{3}{2}$ the estimate

$$
\begin{gathered}
\left\|\nabla p^{k}(t, x)-\nabla p^{k}(t, y)\right\| \leq\left\|\nabla^{2} p^{k}(t)\right\|_{\infty}\|x-y\| \leq \\
C\left[\left\|\gamma^{k}(t)\right\|_{q, l o c}+\left[\gamma^{k}(t)\right]_{1, G}\right]\|x-y\|
\end{gathered}
$$

holds and as a result we obtain

$$
\begin{gathered}
E\left\|\nabla p^{k}\left(\tau, \psi_{t, \tau}^{k}(x)\right)-\nabla p^{k}\left(\tau, \psi_{t, \tau}^{k-1}(x)\right)\right\| \leq \\
C[\beta(\tau)+s(\tau)] E\left\|\psi_{t, \tau}^{k}(x)-\psi_{t, \tau}^{k-1}(x)\right\| .
\end{gathered}
$$

In addition

$$
\begin{gathered}
\left\|\nabla p^{k+1}(t)-\nabla p^{k}(t)\right\|_{\infty} \leq C_{q m}\left[\left\|\gamma^{k+1}(t)-\gamma^{k}(t)\right\|_{q, l o c}+\right. \\
\left.\left\|\gamma^{k+1}(t)-\gamma^{k}(t)\right\|_{m, l o c}\right] \leq C_{q m} \alpha(t)\left[\left\|\nabla u^{k+1}(t)-\nabla u^{k}(t)\right\|_{q, l o c}+\right. \\
\left\|\nabla u^{k}(t)-\nabla u^{k-1}(t)\right\|_{q, l o c}+ \\
\left.\left\|\nabla u^{k+1}(t)-\nabla u^{k}(t)\right\|_{m, l o c}+\left\|\nabla u^{k}(t)-\nabla u^{k-1}(t)\right\|_{m, l o c}\right] .
\end{gathered}
$$

It follows from (3.47) that

$$
\begin{gathered}
n^{k+1}(t, x) \leq C(t)\left[\int_{0}^{t} E\left\|\nabla u^{k}\left(\tau, \psi_{t, \tau}^{k}(x)\right)-\nabla u^{k-1}\left(\tau, \psi_{t, \tau}^{k-1}(x)\right)\right\| d \tau+\right. \\
\left.\int_{0}^{t} n^{k}(\tau, x) d \tau\right]+\int_{0}^{t} \frac{1}{\sigma \sqrt{t-\tau}} C_{1}\left[\left\|\nabla u^{k}(\tau) \nabla u^{k}(\tau)\right\|_{q}+\right. \\
\left.\left\|\nabla u^{k-1}(\tau) \nabla u^{k-1}(\tau)\right\|_{m}\right]^{r}\left(E\left\|\eta^{x, k}(\tau)-\eta^{x, k-1}(\tau)\right\|^{2}\right)^{\frac{1}{2}} d \tau \\
+\int_{0}^{t} \frac{1}{\sigma \sqrt{t-\tau}} e^{\int_{\tau}^{t} \alpha(\theta) d \theta}\left(E\left\|\nabla p^{k+1}\left(\tau, \psi_{t, \tau}^{k}(x)\right)-\nabla p^{k}\left(\tau, \psi_{t, \tau}^{k-1}(x)\right)\right\|^{2}\right)^{\frac{1}{2}} d \tau .
\end{gathered}
$$

By the Hölder inequality we derive that for any positive $f(\tau) \in L_{r, l o c}$ and $\frac{1}{m_{1}}+\frac{1}{r}=1$ and $m_{1}<2$ we have for any compact $G \subset R^{3}$

$$
\int_{K}\left[\int_{0}^{t} \frac{1}{\sigma \sqrt{t-\tau}} f(\tau, x) d \tau\right]^{r} d x \leq \frac{1}{\sigma^{r}} t^{\frac{r\left(2-m_{1}\right)}{2 m_{1}}} \int_{0}^{t} \int_{K} f^{r}(\tau, x) d x d \tau
$$

Then from (3.47) and (3.48) we have for $r>2$

$$
\begin{aligned}
& \zeta_{r}^{k+1}(t) \leq C_{2}(t)\left[\int_{0}^{t} \int_{K}\left[E\left\|u^{k}\left(\tau, \psi_{t, \tau}^{k}(x)\right)-u^{k-1}\left(\tau, \psi_{t, \tau}^{k-1}(x)\right)\right\|^{r} d x d \tau\right]+\right. \\
& \left.\int_{0}^{t} \zeta_{r}^{k}(\tau) d \tau+\int_{0}^{t} \int_{K}\left\|\nabla u^{k-1}\left(\tau, \psi_{t, \tau}^{k}(x)\right)-\nabla u^{k-1}\left(\tau, \psi_{t, \tau}^{k-1}(x)\right)\right\|^{r} d x d \tau\right]
\end{aligned}
$$




$$
\begin{gathered}
+\int_{0}^{t} \frac{1}{\sigma \sqrt{t-\tau}} C\left[\left[\left\|\nabla u^{k}(\tau) \nabla u^{k-1}(\tau)\right\|_{q}+\left\|\nabla u^{k}(\tau) \nabla u^{k-1}(\tau)\right\|_{m}\right]^{r}\right. \\
\left.\int_{K}\left(E\left\|\eta^{x, k}(\tau)-\eta^{x, k-1}(\tau)\right\|^{2}\right)^{\frac{r}{2}} d x\right] d \tau+\int_{0}^{t} \frac{1}{\sigma \sqrt{t-\tau}} e^{\int_{\tau}^{t} \alpha(\theta) d \theta} \\
\int_{K}\left(E\left\|\nabla p^{k+1}\left(\tau, \psi_{t, \tau}^{k}(x)\right)-\nabla p^{k}\left(\tau, \psi_{t, \tau}^{k-1}(x)\right)\right\|^{2}\right)^{\frac{r}{2}} d x d \tau
\end{gathered}
$$

For the function $m_{r}^{k}(t)=\left\|u^{k}(t)-u^{k-1}(t)\right\|_{r, \text { loc }}$ using the apriori estimates proved in lemmas 3.2 -3.8 and theorem 3.9 we obtain

$$
\begin{gathered}
m_{r}^{k+1}(t) \leq C(t)\left[\left(\int_{0}^{t} \int_{K} E\left\|u^{k}\left(\tau, \psi_{t, \tau}^{k}(x)\right)-u^{k-1}\left(\tau, \psi_{t, \tau}^{k-1}(x)\right)\right\|^{r} d x d \tau\right)^{\frac{1}{r}}\right. \\
+\left(\frac{1}{\sigma} t^{\frac{2-m_{1}}{2 m_{1}}} \int_{0}^{t} \int_{K} E \| \nabla u^{k+1}\left(\tau, \psi_{t, \tau}^{k}(x)\right) \nabla u^{k}\left(\tau, \psi_{t, \tau}^{k}(x)\right)-\right. \\
\left.\left.\nabla u^{k-1}\left(\tau, \psi_{t, \tau}^{k}(x)\right) \nabla u^{k-1}\left(\tau, \psi_{t, \tau}^{k-1}(x)\right) \|^{r} d x d \tau\right)^{\frac{1}{r}}\right] \leq \\
C_{1}(t)\left[\left(\int_{0}^{t} m_{r}^{k}(\tau) d \tau\right)^{\frac{1}{r}}+\left(\int_{0}^{t} \int_{K} \alpha(\tau) E\left\|\psi_{t, \tau}^{k}(x)-\psi_{t, \tau}^{k-1}(x)\right\|^{r} d x d \tau\right)^{\frac{1}{r}}+\right. \\
\left.\frac{1}{\sigma} t^{\frac{1}{m_{1}}-\frac{1}{2}}\left(\int_{0}^{t}\left[\rho^{k+1}(\tau)+\rho^{k}(\tau)\right] \zeta_{r}^{k}(\tau) d \tau\right)^{\frac{1}{r}}\right] .
\end{gathered}
$$

Since $u^{k}$ and $\nabla u^{k}$ are proved to be uniformly bounded on $\left[0, T_{1}\right]$ and

$$
\begin{gathered}
\left\|\nabla u^{1}(t, \cdot)-\nabla u_{0}(\cdot)\right\|_{r, l o c} \leq \text { const }<\infty, \\
\left\|u^{1}(t, \cdot)-u_{0}(\cdot)\right\|_{r, l o c} \leq \text { const }<\infty
\end{gathered}
$$

both for $r=m$ and $r=q$ we obtain that there exists a bounded on $\left[0, T_{1}\right]$ positive function $C_{2}(t)$ such that the function $\kappa^{k}(t)=\rho^{k}(t)+\zeta_{m}^{k}(t)+m_{r}^{k}$ satisfies the estimate

$$
\kappa^{k}(t) \leq \frac{\left[C_{2}(t)\right]^{k}}{k !}
$$

and hence $\lim _{k \rightarrow \infty} \kappa^{k}(t)=0$. Since all summands defining $\kappa^{k}(t)$ are positive we deduce that all of them converges to 0 as $k \rightarrow \infty$. As a results we deduce that for each $t \in\left[0, T_{1}\right)$ the family $u^{k}(t, \cdot)$ converges uniformly on compacts and the limiting function $u(t, \cdot) \in \mathbf{C}^{1, \alpha} \cap \mathbf{L}_{m, l o c}$. In addition, we can check that the limiting function $\nabla u(t, x)$ is Lipschitz continuous in $x$. In fact, by lemma 3.8 and theorem 3.9 for each $t \in\left[0, T_{1}\right]$, we have for any $x, y \in G$

$$
\left\|\nabla u^{k}(t, x)-\nabla u^{k}(t, y)\right\| \leq s(t)\|x-y\|,
$$

where $s(t)$ and $T_{1}$ were defined above in lemma 3.8 and the estimate is uniform in $k$.

To prove the uniqueness of the solution of (3.4)-(3.6) constructed above we assume first that there exist two solutions $u_{1}(t, x), u_{2}(t, x)$ to $(3.4)$-(3.6) possessing the same initial data $u_{1}(0, x)=$ $u_{2}(0, x)=u_{0}(x)$. 
Computations similar to those used to prove the convergence of the family $\left(u^{k}(t), \nabla u^{k}(t)\right)$ allow to check that both

$$
\left[\nabla u_{1}(t)-\nabla u_{2}(t)\right]_{\infty}=0 \quad \text { and } \quad\left\|\nabla u_{1}(t)-\nabla u_{2}(t)\right\|_{m, l o c}=0
$$

Finally, we know that a stochastic equation with Lipschitz coefficients has a unique solution of the Cauchy problem. This implies the uniqueness of the solution to (3.4)-(3.6).

Summarizing the above results we see that the following statement is valid.

Theorem 3.10 Assume that $\mathbf{C} 3.2$ holds. Then there exists a unique solution $\psi_{t, x}(s), u(t, x), p(t, x)$ of the system (3.4)-(3.6) for all $t$ from the interval $\left[0, T_{1}\right]$, with $T_{1}$ given by theorem 3.7 and $x \in K$ for any compact $K \subset G$. In addition the process $\psi_{t, x}(s)$ is the Markov process in $R^{3}$ and $u \in$ $\mathbf{C}\left(\left[0, T_{1}\right], \mathbf{C}^{1, \alpha}(K)\right) \cap \mathbf{C}\left(\left[0, T_{1}\right], \mathbf{L}_{q, l o c} \cap \mathbf{L}_{m, l o c}\right)$ for $1<q<\frac{3}{2}<3<m<\infty$.

Proof. First we note that as soon as we know that $u(t, x)$ is locally Lipschitz continuous by classical SDE theory we know that the silution $\psi_{t, 0}(x)$ of the equation (3.4) is the Markov process in $R^{3}$. All other assertions of the theorem are already proved above.

To fulfill our program we have to check that the functions $u(t, x), p(t, x)$ that satisfy $(3.5)$ and (3.6) define a weak solution of (1.5),(1.2).

Let us come back to the Kunita theory of stochastic flows [5], [6] and recall that given a distribution $u_{0} \in \mathcal{D}^{\prime}$ and a stochastic flow $\psi_{t, 0}^{u}$ one can define a stochastic flow $u_{0} \circ \psi_{t, 0}^{u}$ as another distribution satisfying $\left\langle u_{0} \circ \psi_{t, 0}^{u}, h\right\rangle=\left\langle u_{0}, h \circ \phi_{0, t}^{u} J_{0, t}\right\rangle$. Here $\phi_{0, t}^{u}$ is the inverse flow to $\psi_{t, 0}^{u}$. Since any locally integrable function is a distribution, given $u_{0}$ and the solution $\psi_{t, 0}, u(t), p(t)$ of (3.4)-(3.6) constructed above we consider a process $\lambda(t) \in \mathcal{D}^{\prime}$ of the form $\lambda(t)=u_{0}-\int_{0}^{t} \nabla p^{u}(\tau) \circ \phi_{0, \tau}^{u} d \tau$. Next we consider the process

$$
\lambda(t) \circ \psi_{t, 0}^{u}=u_{0} \circ \psi_{t, 0}^{u}-\int_{0}^{t} \nabla p^{u}(\tau) \circ \psi_{t, \tau}^{u} d \tau
$$

and verify that a weak solution $u(t)$ of (3.1) admits the representation $u(t)=E\left[\lambda(t) \circ \psi_{t, 0}^{u}\right]$ and satisfies (3.2).

By the generalized Ito formula we derive

$$
\begin{gathered}
\lambda(t) \circ \psi_{t, 0}^{u}=u_{0}+\int_{0}^{t} \frac{\sigma^{2}}{2} \Delta\left[u(\theta) \circ \psi_{\theta, 0}^{u}\right] d \theta+ \\
\int_{0}^{t} \nabla\left[u(\theta) \circ \psi_{\theta, 0}^{u}\right] \sigma d w(\theta)-\int_{0}^{t} \nabla\left[u(\theta) \circ \psi_{\theta, 0}^{u}\right] u(\theta) d \theta-\int_{0}^{t} \nabla p^{u}(\theta) d \theta,
\end{gathered}
$$

where (3.49) is considered in a weak sense. Hence for $L u=-(u, \nabla) u+\frac{\sigma^{2}}{2} \Delta u$ and the test function $h \in \mathcal{D}$ we have

$$
\begin{gathered}
E\left[\int_{R^{3}} \int_{0}^{t} L\left(u(\tau) \circ \psi_{\tau, s}^{u}(x)\right) d \tau \cdot h(x) d x\right]= \\
E\left[\int_{0}^{t}\left\langle u(\tau) \circ \psi_{\tau, 0}^{u}, L^{*} h\right\rangle d \tau\right]=\int_{0}^{t} L\left\langle E\left[u\left(\tau \circ \psi_{\tau, 0}^{u}\right)\right], h\right\rangle d \tau .
\end{gathered}
$$


As a result we deduce from (3.49) and (3.50)

$$
u(t)=E\left[\lambda(t) \circ \psi_{t, 0}^{u}\right]=u_{0}+\int_{0}^{t} L E\left[u(\tau) \circ \psi_{\tau, 0}^{u}\right] d \tau-\int_{0}^{t} \nabla p^{u}(\tau) d \tau .
$$

Differentiating each term with respect to $t$ we check that the function $u(t)=E\left[\lambda(t) \circ \psi_{t, 0}^{u}\right]$ solves the Cauchy problem (1.1). As soon as the function $p(t)$ was constructed as a solution of the Poisson equation (3.2) we can verify that (1.2) holds as well.

To summarize the obtained results we state the following:

Theorem 3.11. Assume that $\mathbf{C} \mathbf{3 . 2}$ holds. Then the functions $u(t), p(t)$ that solve (3.5),(3.6) satisfy (3.1)-(3.2) in a weak sense for $t \in\left[0, T_{1}\right]$ where $T_{1}$ is defined in theorem 3.9.

Remark 3.13. We have proved that under condition C $\mathbf{3 . 2}$ the system (3.4)-(3.6) gives rise to a weak solution of (3.1)-(3.2). Moreover, when the initial data are smoother, say $u_{0} \in \mathbf{C}^{2, \alpha}, \alpha \in[0,1]$ similar considerations can be applied to verify that the pair $u(t, x), p(t, x)$ given by (3.5)-(3.6) stands for a classical $C^{2}$-smooth solution of (3.1), (3.2).

\section{Lagrangian and stochastic approach to the N-S system}

The probabilistic approach developed in the previous section is in a sense an analogue of the Lagrangian approach developed for the Euler system which coincides with (1.1), (1.2) when $\sigma=0$. The classical Lagrangian path starting at $y$ is governed by the Newton equation

$$
\frac{\partial^{2} \tilde{\phi}_{0, t}(y)}{\partial t^{2}}=F_{\tilde{\phi}}(t, y)
$$

The force $F$ in (4.1) has the form

$$
F_{\tilde{\phi}}(t, y)=-\nabla p\left(t, \tilde{\phi}_{0, t}(y)\right)=-\left[\left(\nabla \tilde{\phi}_{0, t}(y)\right)^{*}\right]^{-1} \nabla\left[p\left(t, \tilde{\phi}_{0, t}(y)\right)\right]
$$

and the incompressibility condition yields $\operatorname{det}\left(\nabla \tilde{\phi}_{0, t}(y)\right)=1$. One can deduce from (4.1) that

$$
\frac{\partial}{\partial t}\left[\frac{\partial \tilde{\phi}_{0, t}^{k}(y)}{\partial t} \frac{\partial \tilde{\phi}_{0, t}^{k}(y)}{\partial y_{i}}\right]=-\frac{\partial q\left(t, \tilde{\phi}_{0, t}(y)\right)}{\partial y_{i}}
$$

where

$$
q(t, y)=p(t, y)-\frac{1}{2}\left\|\frac{\partial \tilde{\phi}_{0, t}(y)}{\partial t}\right\|^{2}
$$

summation over repeated indices is assumed. Integrating (4.3) in time we get

$$
\frac{\partial \tilde{\phi}_{0, t}^{k}(y)}{\partial t} \frac{\partial \tilde{\phi}_{0, t}^{k}(y)}{\partial y_{i}}=u_{0}(y)-\frac{\partial n\left(t, \tilde{\phi}_{0, t}(y)\right)}{\partial y_{i}}
$$

where

$$
n(t, y)=\int_{0}^{t} q(\tau, y) d \tau
$$

and $u_{0}(y)=\left.\frac{\partial \tilde{\phi}_{0, t}(y)}{\partial t}\right|_{t=0}$ is the initial velocity. 
Consider the inverse diffeomorphism $\tilde{\psi}_{t, 0}=\left[\tilde{\phi}_{0, t}\right]^{-1}$, come back to (4.4), multiply it by $\left[\nabla \tilde{\psi}_{t, 0}\right]$ and put $y=\tilde{\psi}_{t, 0}(x)$. As a result we obtain by the chain rule the relation

$$
u^{i}(t, x)=\left(u_{0}^{j}\left(\tilde{\psi}_{t, 0}(x)\right) \nabla_{x_{i}} \tilde{\psi}_{t, 0}^{j}(x)-\int_{0}^{t} \nabla_{x_{i}} q\left(\tau, \tilde{\psi}_{t, \tau}(x)\right) d \tau .\right.
$$

Hence the Euler equations are equivalent to the system consisting of (4.7) and the relation $\Delta n(t, x)=\frac{\partial}{\partial x_{i}}\left\{u_{0}^{k}\left(\tilde{\psi}_{t, 0}(x)\right) \frac{\partial \tilde{\psi}_{t, 0}^{k}(x)}{\partial x_{i}}\right\}$, where $n$ is given by (4.6).

Finally due to divu $=0$ one can rewrite the equation of state (4.7) in the form

$$
u(t)=\mathbf{P}\left\{u_{0}\left(\tilde{\psi}_{t, 0}\right) \nabla \tilde{\psi}_{t, 0}\right\}=\mathbf{P}\left\{\left[\nabla \tilde{\psi}_{t, 0}\right]^{*} u_{0}\left(\tilde{\psi}_{t, 0}\right)\right\},
$$

where $\mathbf{P}=I-\nabla \Delta^{-1} \nabla$ is the Leray projector. The Euler pressure is determined up to additive constants by

$$
p(t, x)=\frac{\partial n(t, x)}{\partial t}+(u(t, x), \nabla) n(t, x)+\frac{1}{2}\|u(t, x)\|^{2} .
$$

When $\sigma \neq 0$ one can develop an analogue of the Lagrange approach as follows. Let us choose $\phi_{0, t}$ to be generated by the stochastic equation

$$
d \phi_{0, \theta}=u\left(\theta, \phi_{0, \theta}\right) d \theta+\sigma d w(\theta), \quad \phi_{0,0}(y)=y,
$$

next set

$$
\psi_{\theta, 0}=\left[\phi_{0, \theta}\right]^{-1},
$$

and finally obtain the closed system by choosing

$$
u(t)=E \mathbf{P}\left[\left(\nabla \psi_{t, 0}\right)\left(u_{0} \circ \psi_{t, 0}\right)\right] .
$$

The system (4.9) - (4.11) was studied by Constantin and Iyer [9], [10]. In [14] the existence and uniqueness of the solution to (4.9) - (4.11) was proved by the successive approximation technique.

The main result due to Constantin and Iyer reads as follows:

Theorem 4.1 Let $k \geq 1$ and $u_{0} \in \mathbf{C}^{k+1, \alpha}$ be divergence free. Then there exists a time interval $[0, T]$ with $T=T\left(k, \alpha, L,\left\|u_{0}\right\|_{k+1, \alpha}\right)$ but independent of viscosity $\sigma$ and a pair $\phi_{0, t}(x), u(t, x)$ such that $u \in C\left([0, T], C^{k+1, \alpha}\right)$ and $(u, \phi)$ satisfy (4.9)-(4.11). Further there exists $U=U\left(k, \alpha, L,\left\|u_{0}\right\|_{k+1, \alpha}\right)$ such that $\|u(t)\|_{k+1, \alpha} \leq U$ for $t \in[0, T]$ and $u$ satisfies the $N$-S system.

Comparing the system (3.4) - (3.6) and the system (4.9) - (4.11) we can check that the process $\psi_{t, 0}$ given by (4.10) has the same distribution as the solution of (3.4). At the other hand the representations for the velocity $u$ and the pressure $p$ in the above systems are different. In the system (3.4) - (3.6) we avoid using the Leray projection and use instead the probabilistic representation of the Poisson equation. This allows us to construct both strong (classical) and weak (distributional) solutions of the Cauchy problem for the N-S system.

At the very end we remark that the approach developed in the previous section does not allow to construct a solution to the Euler system as a limit of the solution to (1.1), (1.2) when $\sigma$ goes to 0, since the appriori estimates in lemma 3.5 and lemma 3.8 cease to be valid.

Acknowledgement. The authors gratefully acknowledge the financial support of DFG Grant 436 RUS 113/823.

Received: February 2009. Revised: March 2009. 


\section{References}

[1] S. Albeverio and Ya. Belopolskaya, Probabilistic approach to hydrodynamic equations. In the book Probabilistic Methods in Hydrodynamics. World Scientific (2003) 1-21.

[2] Y. Le Jan and A. Sznitman, Stochastic cascades and 3-dimensional Navier Stokes equations, Prob. Theory Relat. Fields, 109 (1997) 343-366.

[3] M. Ossiander, A probabilistic representation of solution of the incompressible Navier-Stokes equations in $R^{3}$. Prob. Theory Relat. Fields, 1332 (2005) 267-298.

[4] B. Busnello, F. Flandoli and M. Romito, A probabilistic representation for the vorticity of a $3 D$ viscous fluid and for general systems of parabolic equations, Proc. Edinburgh Math. Soc., 48 2 (2005) 295-336.

[5] H. Kunita, Stochastic flows acting on Schwartz distributions. J. Theor. Pobab. 72 (1994) 247-278.

[6] H. Kunita, Generalized solutions of stochastic partial differential equations. J. Theor. Pobab.7 2 (1994) 279-308.

[7] Ya. Belopolskaya and Yu. Dalecky, Investigation of the Cauchy problem for systems of quasilinear equations via Markov processes . Izv VUZ Matematika. N 12 (1978) 6-17.

[8] Ya. Belopolskaya and Yu. Dalecky, Stochastic equations and differential geometry. Kluwer (1990).

[9] P. Constantin, An Eulerian-Lagrangian approach to the Navier-Stokes equations. Commun. Math. Phys. bf 216 (2001) 663-686.

[10] P. Constantin And G. Iyer, A stochastic Lagrangian representation of the 3-dimensional incompressible Navier-Stokes equations. Commun. Pure and Appl. Math. 613 (2007) 330 - 345.

[11] P.G. Lemarie-Rieusset, Recent Developments in the Navier-Stokes Problem, Chapman\&Hall, CRC (2002).

[12] K. D. Elworthy And Xue-Mei Li, Differentiation of heat semigroups and applications, J. Funct. Anal. 125, No.1, (1994) 252-286.

[13] D. Gilbarg And N.S.Trudinger, Elliptical partial differential equations, second eddition. Springer-Verlag Berlin Heidelberg New York Tokyo (1983).

[14] G. A. IYER, stochastic Lagrangian formulation of the incompressible Navier-Stokes and related transport equations. PhD dissertation Dept. Math. Univ. Chicago (2006). 